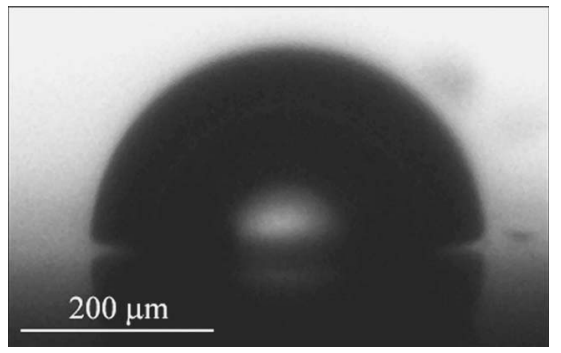

FIG. 1. Side view.

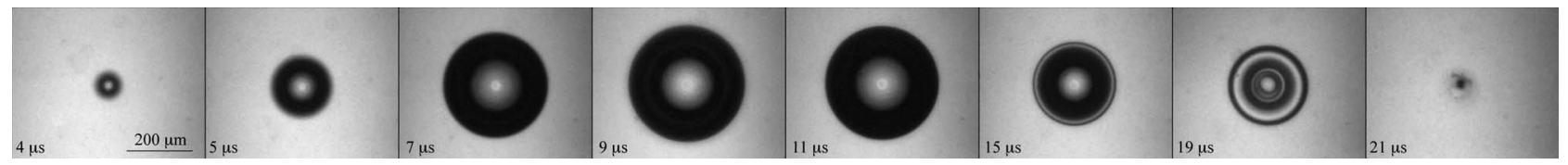

FIG. 2. Single bubble.

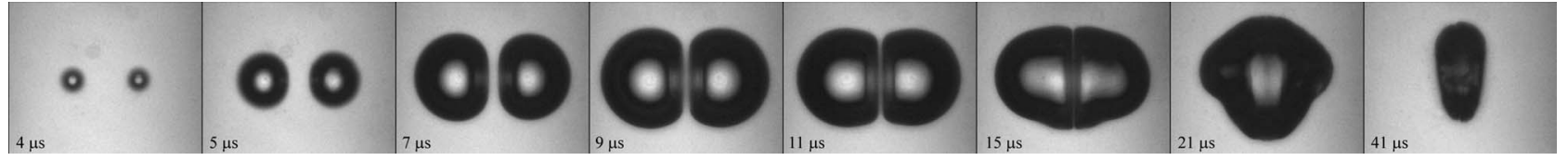

FIG. 3. Two bubbles.

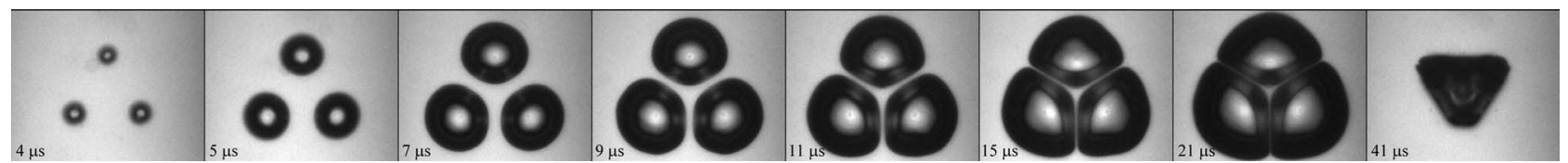

FIG. 4. Three bubbles.
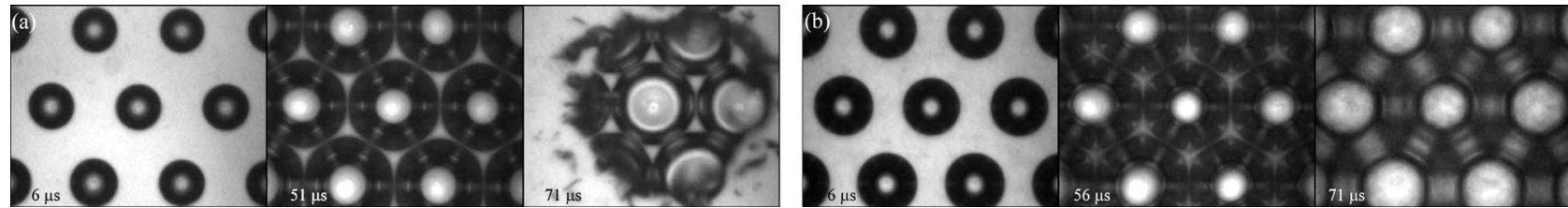

FIG. 5. Hexagonal pattern of bubbles.

\section{Cavitating bubbles on patterned surfaces}

Nicolas Bremond, Manish Arora, Claus-Dieter Ohl, and Detlef Lohse

Physics of Fluids, University of Twente, 7500 AE Enschede, The Netherlands

(Received 8 March 2005; published online 26 August 2005)

[DOI: 10.1063/1.1942514]

We present an experimental investigation of cavitation on solid surfaces induced by lowering the liquid pressure. For this purpose, microcavities are etched on a silicon plate which is coated by a hydrophobic layer. These cavitation nuclei are then subjected to a negative pressure pulse of $-4 \mathrm{MPa}$ for a duration of $3 \mu \mathrm{s}$. The time $t=0$ is defined through the pressure wave front reaching the location of the cavities. The dynamics of the bubbles is visualized and recorded through an inverted microscope $(20 \times)$ with a charge coupled device camera. Motion blurring is minimized by a short illumination time of $0.2 \mu \mathrm{s}$.

As shown in Fig. 1, a single bubble keeps a semispherical shape during expansion. We note the presence of a thin liquid film between the bubble and the solid surface which might result from the pinning of the triple contact line at the microcavity. The bubble reaches a maximal size and then quickly collapses (Fig. 2). If bubbles grow close to each other, they lose their semispherical shape and can eventually coalesce as shown in Fig. 3 for two bubbles and in Fig. 4 for three bubbles, which are initially $200 \mu \mathrm{m}$ apart from one another. Note that due to shielding the collapse for multibubble systems lasts longer than for an isolated bubble.

Two examples of the interaction of bubbles set on a hexagonal lattice are presented in Fig. 5. The pitch of the pattern is $200 \mu \mathrm{m}$ for both sequences, the left pattern is subjected to $-4 \mathrm{MPa}$, and the right to $-7 \mathrm{MPa}$. The first experiment exhibits individual collapses whereas multicoalescence is observed for a larger negative pressure pulse. 